\title{
Anticancer effects of dietary administration of secoisolariciresinol diglucoside in a patient of gastrointestinal stromal tumor: a case report
}

\author{
Hao Wu, MD, MS ${ }^{a, b, c, d, e, f}$, Yao Wang, BS ${ }^{a, d, e}$, Jia-Tao Chen, BS ${ }^{a, d, e}$, Li-Ping Wang, MD ${ }^{a, g}$, Gui-Rong Liu, BS ${ }^{a, d, e}$, \\ Shu-Lin Liu, MD, PhD ${ }^{a, d, e, f, t}$,
}

\begin{abstract}
Introduction: Gastrointestinal stromal tumor (GIST) is among the leading malignancies of the digestive system. GIST is not susceptible to chemotherapies and is prone to recurrence or metastasis after surgical operation. Secoisolariciresinol diglucoside (SDG) was suggested to have potential anticancer effects, but no clinical evidence had been available. Here we report successful treatment of a postoperative GIST case with dietary SDG.

Presentation of Case: The patient was a 58-year-old man. He had GIST and received resection of small intestinal lesions 1 year before. He was diagnosed with postoperation of GIST and presented with high level of serum carbohydrate antigen 72-4 (CA72-4). The patient was then treated with dietary administration of SDG with his voluntary acceptance for 5 months, and underwent 2 serum CA72-4 measurements during this period. CA72-4 level of the patient was restored to normal range after treatment with dietary SDG for 1 month. There was no aberrant CA72-4 level, recurrence or metastasis after the treatment with dietary SDG.

Discussion: This is to our knowledge the first report on application of dietary SDG on a postoperative GIST patient with aberrant level of serum CA72-4. SDG can be transformed into active substances with antitumor effects by human gut bacteria. Dietary SDG might inhibit tumorigenicity and malignant behavior of GIST cells.
\end{abstract}

Conclusion: The excellent effects suggest dietary SDG to be a potential therapy for GIST, especially against recurrence or metastasis.

Keywords: GIST, CA72-4, Dietary SDG, Recurrence, Metastasis, Case report

\section{Key points}

(1) Dietary secoisolariciresinol diglucoside (SDG) shows potent anticancer effect reflected by complete patient recovery.

${ }^{a}$ Genomics Research Center (State-Province Key Laboratories of BiomedicinePharmaceutics of China), College of Pharmacy, Harbin Medical University, ${ }^{b}$ Sino-Russian Medical Research Center, Harbin Medical University Cancer Hospital, cHeilongjiang Academy of Medical Sciences, ${ }^{d H M U-U C C S M}$ Centre for Infection and Genomics, ${ }^{e}$ Translational Medicine Research and Cooperation Center of Northern China, Heilongjiang Academy of Medical Sciences, Harbin, China, ${ }^{f}$ Department of Microbiology, Immunology and Infectious Diseases, University of Calgary, Calgary, AB, Canada and IKangyuan Hospital, 9 Tongfatoudao Street, Harbin, China

This manuscript has been peer reviewed.

Sponsorships or competing interests that may be relevant to content are disclosed at the end of this article.

*Corresponding author. Address: Genomics Research Center, Harbin Medical University, Harbin 150081, China. Tel: + 8645186692236; fax: + 8645187086735. E-mail address: slliu@hrbmu.edu.cn (S.-L. Liu).

Copyright $@ 2021$ The Authors. Published by Wolters Kluwer Health, Inc. on behalf of IJS Publishing Group Ltd. This is an open-access article distributed under the terms of the Creative Commons Attribution-Non Commercial-No Derivatives License 4.0 (CCBY-NC-ND), where it is permissible to download and share the work provided it is properly cited. The work cannot be changed in any way or used commercially without permission from the journal.

International Journal of Surgery Oncology (2021) 5:e103

Received 3 August 2020; Accepted 16 September 2020

Published online 20 January 2021

Supplemental Digital Content is available for this article. Direct URL citations appear in the printed text and are provided in the HTML and PDF versions of this article on the journal's website, www.IJSOncology.com.

http://dx.doi.org/10.1097//J9.00000000000000103
(2) Carbohydrate antigen 72-4 (CA72-4) level becoming normal correlated very well with the recovery of the patient.

(3) SDG is easily edible and may be used widely for cancer prevention and treatment.

\section{Introduction}

Gastrointestinal stromal tumor (GIST) is one of the most common mesenchymal malignancies in the gastrointestinal tract, with no specific clinical manifestations to be used for diagnosis at early stages ${ }^{[1]}$. The incidence rate of GIST is $~ 0.001 \%-0.0015 \%$ but a trend of increase is obvious in recent years ${ }^{[2]}$. GIST is mostly seen in men over 50 years old, occurring in the stomach $(60 \%-70 \%)$, the small intestine $(25 \%-36 \%)$, colon $(5 \%)$ or esophagus $(5 \%)^{[3]}$. About $10 \%-30 \%$ of the GIST patients develop metastasis ${ }^{[4]}$, most commonly in the liver $(65 \%)$, followed by the peritoneum $(21 \%)$, the bone $(10 \%)$, and lung $(10 \%)^{[5]}$. The treatment for GIST is quite challenging, as GIST is not susceptible to radiotherapy or chemotherapy. As a result, surgical resection is almost the only method left to treat the disease. However, over half of the patients would face recurrence or metastasis after the surgical operation ${ }^{[6]}$. Multiple genetic changes have been identified for their associations with GIST, including C-KIT mutations in $70 \%-80 \%$ and PDGFR- $\alpha$ mutations in $5 \%-10 \%$ of the examined cases. The downstream signaling pathways of C-KIT include PI3K/ART, Src family kinases, RAS-ERK, and JAKSTAT $^{[7]}$.

Currently, tyrosine kinase inhibitors (TKIs) as routine targeted drugs $^{[8]}$, with Imatinib as a representative, are often used after GIST surgery. Imatinib can inhibit the activities of both C-KIT 
and PDGFR- $\alpha^{[9]}$. However, C-KIT/PDGFRA- $\alpha$ gene mutations may lead to drug resistance and cause increasing relapse rate of GIST. Most GIST patients treated with Imatinib may develop drug resistance within 2 years $^{[10,11]}$, complicating the situations that about $14 \%$ of the patients with GISTs have primary resistance to Imatinib ${ }^{[12]}$. Sunitinib and Regorafenib are second-line and third-line drugs for the treatment of Imatinib-resistant patients, respectively, but patients are still prone to drug resistance after treatment for a few months ${ }^{[13,14]}$. In addition, a type of GIST, called wild-type GIST, does not have mutations in the C-KIT or PDGFR- $\alpha$ gene ${ }^{[15]}$ and so is naturally resistant to the imatinib treatment. While long-term use of imatinib may be accompanied with side effects such as fatigue, diarrhea, nausea, periorbital edema, muscle cramps, and skin rash, etc ${ }^{[16]}$, the emergence of drug resistance and the high cost of treatment are even more problematic ${ }^{[13]}$. This situation calls for novel therapeutics to minimize the adverse reactions and maximize treatment effects.

SDG, a kind of phytolignans existing in a broad range of plants, with the seed of flax (Linum usitatissimum L) being among the richest sources, has strong bioactivities, including antioxidant, antidepression, and anticancer effects, and may also affect lipid regulation and cholesterol metabolism ${ }^{[17-21]}$. Here we report a GIST case treated with plant materials containing SDG after surgical removal of the tumor, because the patient refused to accept any chemotherapy. The patient achieved remarkable treatment effects as reflected by rapid recovery of his general health status and drastically lowered CA72-4 level (> $300 \mathrm{U} / \mathrm{mL}$ before the use of the dietary SDG and $3.99 \mathrm{U} / \mathrm{mL}$ after the use of the dietary SDG), suggesting that the dietary SDG had potent suppressive activities on GIST.

\section{Case presentation}

\section{Medical records}

The patient was a 56-year-old man, who complained of intermittent hematochezia lasting for over 1 month. He was born in Inner Mongolia Autonomous Region, China. He is of Han ethnic group

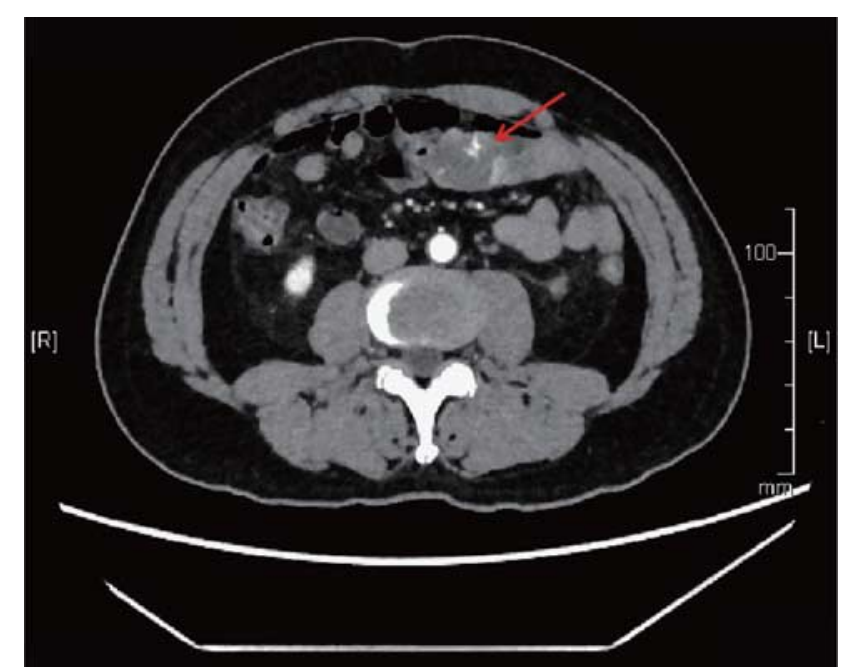

Figure 1. Abdominal enhancement computed tomography showed that there was a jejunal mass (red arrow, high-density) in region of the left abdomen. and retired. The patient's medical history included gastric bleeding and he underwent subtotal gastrectomy 35 years before. He had no history of hypertension, diabetes, cardiovascular or mental diseases and no history of allergy, smoking, drinking or drug use. His spouse was healthy. He denied a history of familial hereditary diseases. The physical examination of the patient showed that the abdomen was flat and soft, the abdominal breathing and intestinal sound were normal. Percussion of the abdomen was a drum sound. No abnormality was found on anal examination. There was no abdominal mass, subcutaneous varicose vein of abdominal wall, gastrointestinal type, peristaltic wave, rebound pain, muscle tension, shifting dullness, gurgling or Murphy sign. Liver and spleen were impalpable. The patient's general conditions, including the mental, diet, sleep, and urination states, were normal. No nausea or vomiting was observed. The abdominal enhancement computed tomography (CT) showed that there was a jejunal mass in the left abdomen, suggesting neoplastic lesions (Fig. 1). The patient underwent laparoscopic exploration and was then treated with small intestinal tumor resection operation. The operation was performed by the chief physician of the General Surgery Department and the procedure went smoothly. Postoperative pathology showed that the tumor of the small intestine was spindle cell type, consistent with the diagnosis of GIST according to immunohistochemistry (IHC). The volume of the tumor was $3.4 \times 3.2 \times 2.8 \mathrm{~cm}$. The mitosis counts were $<5 / 50 \mathrm{HPF}$. There was extensive hemorrhage and necrosis in the tissue. The IHC results of some key molecules were as follows: CD117 $(+)$, DOG-1 $(+)$, Desmin (-), PDGFR-a (+), S-100 (- ), $\alpha$-SMA (-), Ki67 (MIB-1) $(2 \%+), \mathrm{CK}(\mathrm{AE} 1 / \mathrm{AE} 3)(-)$, vimentin $(+), \mathrm{CD} 34(+), \mathrm{D} 2-40(+)$, and CD31 (-) (Figs. 2A-M). The patient recovered well after the operation. The discharge diagnosis of the patient was gastrointestinal bleeding and GIST.

\section{Postoperative reexamination and dietary administration of SDG}

The patient did not receive any other treatment after the surgical operation. He had another examination of serum tumor markers and a CT scan of the abdomen 1 year after GIST resection. The CT results showed no abnormalities around the abdomen. Serum levels of detected biomarkers were as follows: AFP, $2.68 \mathrm{ng} / \mathrm{mL}$ (reference value: $0-7.00 \mathrm{ng} / \mathrm{mL}$ ); CEA, $1.60 \mathrm{ng} / \mathrm{mL}$ (reference value: $0-5 \mathrm{ng} / \mathrm{mL}$ ); CA125, $19.70 \mathrm{U} / \mathrm{mL}$ (reference value: $0-35$ $\mathrm{U} / \mathrm{mL}$ ); CA-199, $6.50 \mathrm{U} / \mathrm{mL}$ (reference value: $0-27 \mathrm{U} / \mathrm{mL}$ ); and CA-153, $10.12 \mathrm{U} / \mathrm{mL}$ (reference value: $0-25 \mathrm{U} / \mathrm{mL}$ ). However, the serum level of carbohydrate antigen (CA72-4) was $>300 \mathrm{U} / \mathrm{mL}$ (the upper limit of detection; reference value: $0-6.9 \mathrm{U} / \mathrm{mL}$ ) (Supplement Fig. 1, Supplemental Digital Content 1, http://links. lww.com/IJSO/A14), indicating possible recurrence or metastasis of postoperative GIST or other gastrointestinal tumors. As the patient refused to receive any chemotherapy, we treated him with dietary SDG, $15 \mathrm{~g}$ a dose twice a day, in the morning and at night. He was told to pay attention to his general condition and to have CA72-4 reexamined on a monthly basis. The patient continued this treatment and did not receive any other medical intervention. He had CA72-4 re-checked after one month. Remarkably, the level was 1.39 U/mL (Supplement Fig. 2, Supplemental Digital Content 1, http://links.lww.com/IJSO/A14). He continued to take dietary SDG and had CA72-4 tested again 3 months after the previous reexamination and it was $3.99 \mathrm{U} / \mathrm{mL}$ (Supplement Fig. 3, Supplemental Digital Content 1, http://links.lww.com/IJSO/A14). 

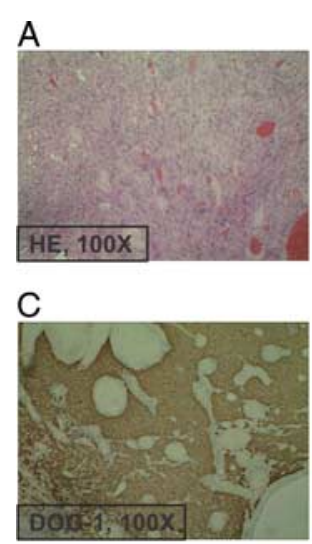

E

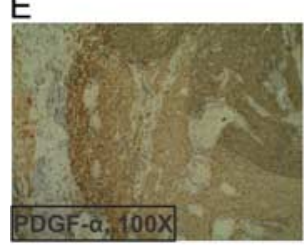

G

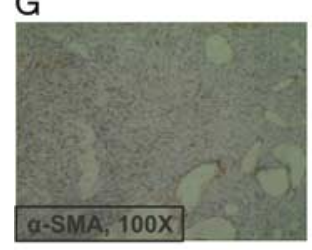

I

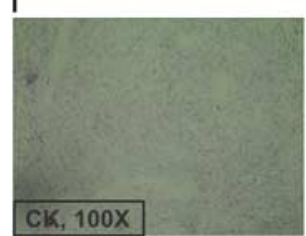

$\mathrm{K}$

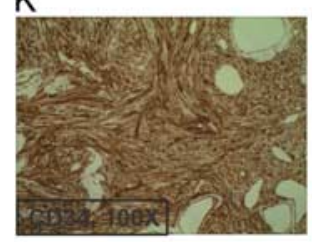

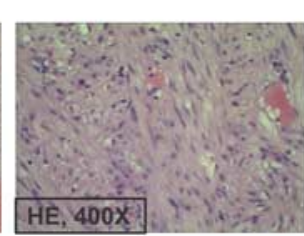
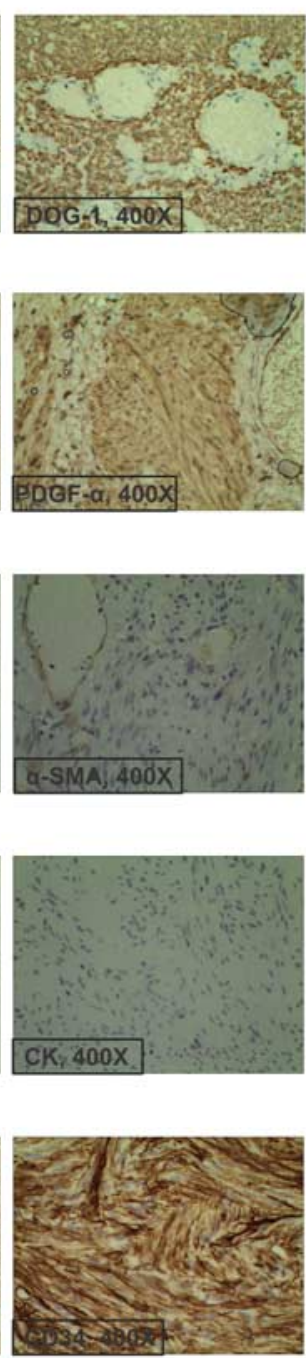

M

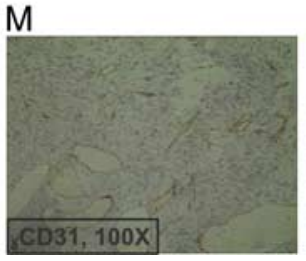

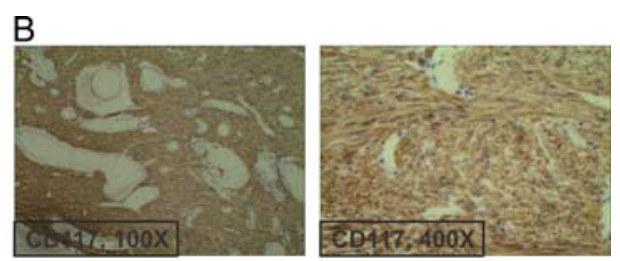

$\mathrm{D}$
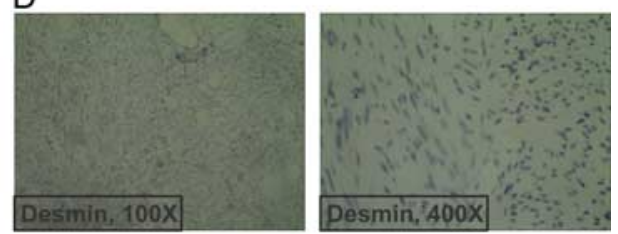

$\mathrm{F}$
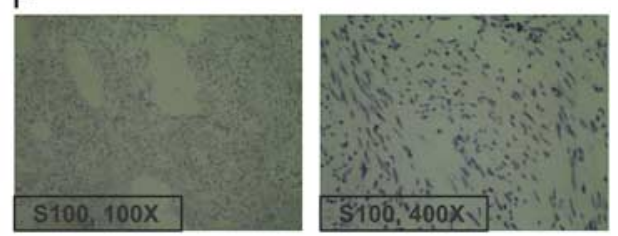

$\mathrm{H}$
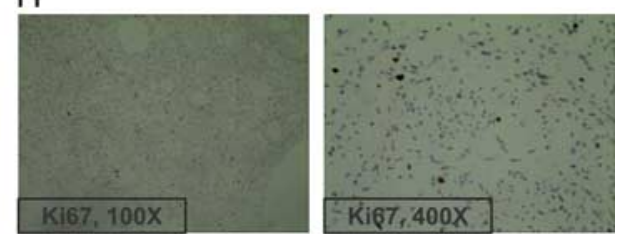

J
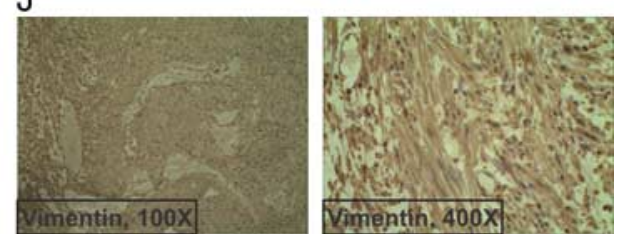

L
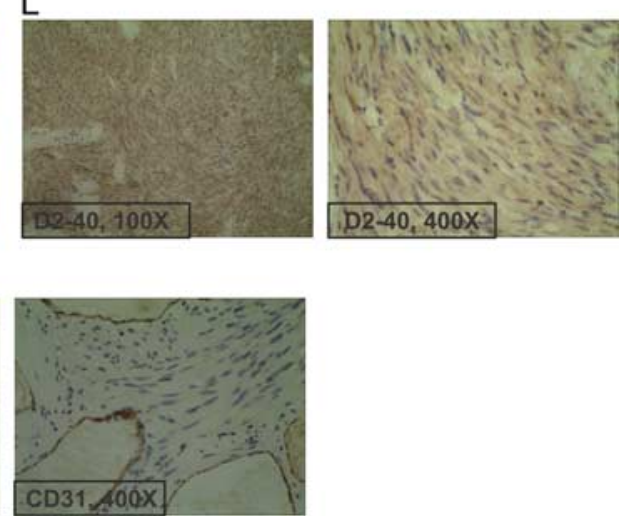

Figure 2. Representative hematoxylin and eosin $(\mathrm{HE})$ and immunohistochemistry $(\mathrm{IHC})$ images. A, Representative $\mathrm{HE}$ staining images. B, Representative $I \mathrm{HC}$ images of CD117. C, Representative IHC images of DOG-1. D, Representative IHC images of Desmin. E, Representative IHC images of PDGFR- $\alpha$. F, Representative IHC images of S-100. G, Representative IHC images of $\alpha$-SMA. H, Representative IHC images of Ki67. I, Representative IHC images of CK. J, Representative $\mathrm{IHC}$ images of vimentin. K, Representative $\mathrm{HC}$ images of CD34. L, Representative $I H C$ images of D2-40. M, Representative IHC images of CD31.

During the period of dietary SDG administration, the patient kept reporting good physical conditions, indicating a good tolerability with dietary SDG intervention in addition to the excellent therapeutic effects. He felt much stronger and regained normal routine activities including aerobics, better mood, and good sleep quality. This case is reported in accordance with the SCARE 2018 standard $^{[22]}$, and additional details can be found in the full SCARE list. 


\section{Discussion}

Numerous mutations and high heterogeneity of GIST facilitate drug-resistance development during targeted therapy applications and therefore call for novel therapeutics ${ }^{[1]}$. CA72-4 is a glycoprotein antigen that is highly expressed in a variety of tumors including GIST $^{[23,24]}$. We treated this postoperative GIST patient with dietary SDG and achieved general recovery of the patient from the malignancy, with greatly improved health status. Imporatantly, the drastically increased CA72-4 level suggests tumor recurrence or metastasis soon after the surgery and the rapid drop of the CA72-4 level to the normal range reflects effective suppression of the tumor recurrence or metastasis associated with the use of dietary SDG.

We gained several learning points in the treatment of this case with dietary SDG. First, no metastasis was found in the regional lymph node of the patient when he received surgery operation, indicating that he had a relatively low aggressive type of GIST. However, CA72-4 level increased abnormally one year after the operation, suggesting that even low-aggressive GIST may have the possibility of tumor recurrence or metastasis. Therefore, it seems improper to decide whether or not to carry out targeted treatment after operation based solely on the level of risk assessment. Second, considering the side effects of targeted drugs against GIST ${ }^{[16]}$, it is not appropriate to use them for prophylactic medication. This led to our way of thinking for preventing the recurrence or metastasis of a low-aggressive GIST patient that has no indication of targeted therapy after surgery operation. In this case, it is extremely intriguing that the CA72-4 level of this patient returned to normal level one month after the dietary SDG treatment and sustained a normal range with no recurrence or metastasis, demonstrating the promising use of dietary SDG against GIST. Furthermore, the patient did not show any side effects and regained active routine life.

The possible mechanism by which SDG exerts the anticancer effects seems to be through the biotransformation of SDG into enterodiol and enterolactone (ENL) by gut bacteria ${ }^{[18-20]}$. Our and others' previous work demonstrates that enterodiol and ENL reduce the metastasis of female malignant tumor cells by inhibiting the adhesion, invasion and motility and downregulate the expression of MMP-2, MMP-9 and MMP-14 ${ }^{[25,26]}$. Moreover, treating mice with SDG or ENL inhibited the secretion of IL-1 $\beta$ in stroma around cancer tissues ${ }^{[27]}$. IL-1 $\beta$ has been proved to promote tumorigenicity and peripheral angiogenesis of tumor cells $^{[28]}$. Therefore, we consider that dietary SDG may decrease the CA72-4 level of this patient through these mechanisms. Further investigation is needed to experimentally explore the inhibitory effects of SDG on GIST.

In short, this case suggests that dietary SDG is a potential anticancer therapy.

\section{Consent for publication}

Written informed consent was obtained from the patient for publication of this case report and accompanying materials. A copy of the written consent is available for review by the Editorin-Chief of this journal on request.

\section{Ethical approval}

Written informed consent for publication was obtained from the patient.

\section{Sources of funding}

Supported by: (1) National Natural Science Foundation of China, GuiRong Liu, Grant Numbers: 81971910. (2) National Natural Science Foundation of China, Shu-Lin Liu, Grant Numbers: 81671980. (3) National Natural Science Foundation of China, Shu-Lin Liu, Grant Numbers: 81871623. (4) Heilongiiang Health and Family Planning Commission Foundation, Hao Wu, Grant Numbers: 2019-056. (5) Haiyan Fund Project of Harbin Medical University Cancer Hospital, Hao Wu, Grant Numbers: JJQN 2018-06.

\section{Author contribution}

S.-L.L. and H.W.: conceptualization. H.W., Y.W., J.-T.C., and S.-L.L.: investigation. S.-L.L., L.-P.W., and G.-R.L.: project administration. L.-P.W.: resources. Y.W. and J.-T.C.: software. H.W. and S.-L.L.: validation.

\section{Conflict of interest disclosures}

The authors declare that they have no financial conflict of interest with regard to the content of this report.

\section{Research registration unique identifying number (UIN)}

Not applied.

\section{Guarantor}

Shu-Lin Liu.

\section{Acknowledgments}

The authors thank the patient for his cooperation. They also acknowledge all the research staff for their contributions to this project. The authors thank Ding Nan for taking pictures of HE and IHC. The authors also thank Bo-Lin Wu for selecting representative CT image.

\section{References}

[1] Li J, Ye Y, Wang J, et al. Chinese consensus guidelines for diagnosis and management of gastrointestinal stromal tumor. Chin J Cancer Res 2017; 29:281-93.

[2] Demetri GD, von Mehren M, Antonescu CR, et al. NCCN Task Force report: update on the management of patients with gastrointestinal stromal tumors. J Natl Compr Canc Netw 2010;8(suppl 2):S1-41; quiz S42-4.

[3] Liu Z, Tian Y, Liu S, et al. Clinicopathological feature and prognosis of primary hepatic gastrointestinal stromal tumor. Cancer Med 2016;5:2268-75.

[4] Supsamutchai C, Wilasrusmee C, Hiranyatheb P, et al. A cohort study of prognostic factors associated with recurrence or metastasis of gastrointestinal stromal tumor (GIST) of stomach. Ann Med Surg (Lond) 2018; $35: 1-5$.

[5] Salame H, Issa M, Nicolas G, et al. A rare case of a ruptured metastatic hepatic lesion from a jejunal gastrointestinal stromal tumor (GIST) treated by arterial embolization. Am J Case Rep 2018;19:1480-7.

[6] Zhu CZ, Liu D, Kang WM, et al. Ghrelin and gastrointestinal stromal tumors. World J Gastroenterol 2017;23:1758-63.

[7] Jakhetiya A, Garg PK, Prakash G, et al. Targeted therapy of gastrointestinal stromal tumours. World J Gastrointest Surg 2016;8:345-52.

[8] Seifert AM, Zeng S, Zhang JQ, et al. PD-1/PD-L1 blockade enhances Tcell activity and antitumor efficacy of imatinib in gastrointestinal stromal tumors. Clin Cancer Res 2017;23:454-65. 
[9] Reichardt P. The story of imatinib in GIST-a journey through the development of a targeted therapy. Oncol Res Treat 2018;41:472-7.

[10] Blanke CD, Rankin C, Demetri GD, et al. Phase III randomized, intergroup trial assessing imatinib mesylate at two dose levels in patients with unresectable or metastatic gastrointestinal stromal tumors expressing the kit receptor tyrosine kinase: S0033. J Clin Oncol 2008;26:626-32.

[11] Verweij J, Casali PG, Zalcberg J, et al. Progression-free survival in gastrointestinal stromal tumours with high-dose imatinib: randomised trial. Lancet 2004;364:1127-34.

[12] Vadakara J, von Mehren M. Gastrointestinal stromal tumors: management of metastatic disease and emerging therapies. Hematol Oncol Clin North Am 2013;27:905-20.

[13] Demetri GD, Reichardt P, Kang YK, et al. Efficacy and safety of regorafenib for advanced gastrointestinal stromal tumours after failure of imatinib and sunitinib (GRID): an international, multicentre, randomised, placebo-controlled, phase 3 trial. Lancet 2013;381:295-302.

[14] Demetri GD, van Oosterom AT, Garrett CR, et al. Efficacy and safety of sunitinib in patients with advanced gastrointestinal stromal tumour after failure of imatinib: a randomised controlled trial. Lancet 2006;368:1329-38.

[15] Nannini M, Biasco G, Di Scioscio V, et al. Clinical, radiological and biological features of lung metastases in gastrointestinal stromal tumors (case reports). Oncol Rep 2011;25:113-20.

[16] Sodergren SC, White A, Efficace F, et al. Systematic review of the side effects associated with tyrosine kinase inhibitors used in the treatment of gastrointestinal stromal tumours on behalf of the EORTC Quality of Life Group. Crit Rev Oncol Hematol 2014;91:35-46.

[17] Socrier L, Quéro A, Verdu M, et al. Flax phenolic compounds as inhibitors of lipid oxidation: Elucidation of their mechanisms of action. Food Chem 2019;274:651-8

[18] Wang CZ, Ma XQ, Yang DH, et al. Production of enterodiol from defatted flaxseeds through biotransformation by human intestinal bacteria. BMC Microbiol 2010;10:115.
[19] Tao YL, Yang DH, Zhang YT, et al. Cloning, expression, and characterization of the $\beta$-glucosidase hydrolyzing secoisolariciresinol diglucoside to secoisolariciresinol from Bacteroides uniformis ZL1. Appl Microbiol Biotechnol 2014;98:2519-31.

[20] Liu H, Liu J, Wang S, et al. Enterolactone has stronger effects than enterodiol on ovarian cancer. J Ovarian Res 2017;10:49.

[21] Zhou YJ, Zhao DD, Liu H, et al. Cancer killers in the human gut microbiota: diverse phylogeny and broad spectra. Oncotarget 2017;8:49574-91.

[22] Agha RA, Borrelli MR, Farwana R, et al. The SCARE 2018 statement: updating consensus surgical case report (SCARE) guidelines. Int J Surg 2018; 60:132-6.

[23] Park K, Jang G, Baek S, et al. Usefulness of combined PET/CT to assess regional lymph node involvement in gastric cancer. Tumori 2014;100: 201-6.

[24] Gwak HK, Lee JH, Park SG. Preliminary evaluation of clinical utility of CYFRA 21-1, CA 72-4, NSE, CA19-9 and CEA in stomach cancer. Asian Pac J Cancer Prev 2014;15:4933-8.

[25] Power KA, Ward WE, Chen JM, et al. Mammalian lignans enterolactone and enterodiol, alone and in combination with the isoflavone genistein, do not promote the growth of MCF-7 xenografts in ovariectomized athymic nude mice. Int J Cancer 2006;118:1316-20.

[26] Mali AV, Wagh UV, Hegde MV, et al. In vitro anti-metastatic activity of enterolactone, a mammalian lignan derived from flax lignan, and downregulation of matrix metalloproteinases in MCF-7 and MDA MB 231 cell lines. Indian J Cancer 2012;49:181-7.

[27] Lindahl G, Saarinen N, Abrahamsson A, et al. Tamoxifen, flaxseed, and the lignan enterolactone increase stroma- and cancer cell-derived IL-1Ra and decrease tumor angiogenesis in estrogen-dependent breast cancer. Cancer Res 2011;71:51-60.

[28] Nakao S, Kuwano T, Miyahara TM, et al. Infiltration of COX-2expressing macrophages is a prerequisite for IL-1 beta-induced neovascularization and tumor growth. J Clin Invest 2005;115:2979-91. 